\title{
ASYMPTOTIC BEHAVIOR OF NONOSCILLATORY SOLUTIONS OF A HIGHER ORDER FUNCTIONAL DIFFERENTIAL EQUATION
}

\section{HIROSHI ONOSE}

\author{
The asymptotic behavior of nonoscillatory solutions of $n$th \\ order nonlinear functional differential equations

$$
\begin{aligned}
\left(r _ { n - 1 } ( t ) \left(r_{n-2}(t)\left(\ldots\left(r_{2}(t)\left(r_{1}(t) y^{\prime}(t)\right)^{\prime}\right)^{\prime}\right)\right.\right. & \left.\left.\ldots)^{\prime}\right)^{\prime}\right) \\
& +a(t) f(y(g(t)))=b(t)
\end{aligned}
$$

is investigated. Sufficient conditions are provided which ensure that all nonoscillatory solutions approach zero as $t \rightarrow \infty$.

\section{Introduction}

We consider the $n$th order functional differential equation with deviating argument

$$
\text { (1) } \begin{aligned}
\left.\left.\left(r_{n-1}(t)\left(r_{n-2}(t)\left(\ldots\left(r_{2}(t)\left(r_{1}(t) y^{\prime}(t)\right)\right)^{\prime}\right) ' \ldots\right)^{\prime}\right)\right)^{\prime}\right) ' & \\
& +a(t) f(y(g(t)))=b(t),
\end{aligned}
$$

where $a(t), b(t), g(t), r_{1}(t), \ldots, r_{n-1}(t)$ are real-valued and continuous on $[T, \infty)$ and $f(y)$ is real-valued and continuous on $(-\infty, \infty)$.

The following conditions are assumed to hold throughout the paper:

(2a) $\lim _{t \rightarrow \infty} g(t)=\infty ;$
(2b) $y f(y)>0$ for $y \neq 0$;

Received 23 February 1981. 
(2c) $r_{i}(t)>0$ and $\lim _{t \rightarrow \infty} \rho_{i}(t)=0$, where $\rho_{i}(t)=\int_{t}^{\infty} \frac{\rho_{i-1}(s)}{r_{i}(s)} d s$, $i=1, \ldots, n-1,\left(\rho_{0}(t) \equiv 1\right)$.

We note that the condition (2c) is satisfied if

$$
\int_{T}^{\infty} \frac{d t}{r_{i}(t)}<\infty, i=1, \ldots, n_{i}-1 .
$$

We restrict our consideration to those solutions $y(t)$ of (1) which exist on some ray $\left[T_{y}, \infty\right)$ and satisfy

$$
\sup \left\{|y(t)|: t_{0} \leq t<\infty\right\}>0
$$

for any $t_{0} \in\left[T_{y}, \infty\right)$. Such a solution is said to be oscillatory if it has arbitrary large zeros; otherwise, it is said to be nonoscillatory. It is important to find sufficient conditions in order that all nonoscillatory solutions of ( 1 ) tend to zero as $t \rightarrow \infty$. Many authors have studied this problem, for example, Hammett [3], Graef and Spikes [1], Grimmer [2], Kartsatos [4], Kusano and Onose ([5], [6]), Londen [7] and Singh [8]. In this paper we present some results on this problem.

\section{Non-oscillation theorems}

We use the following lemmas to prove our results.

LEMMA 1 [5]. Consider the differential equation

$$
u^{\prime}(t)-\frac{\rho^{\prime}(t)}{\rho(t)} u(t)+\frac{\rho^{\prime}(t)}{\rho(t)} \phi(t)=0
$$

where $\phi(t)$ is continuous on $[T, \infty), \rho(t)$ is continuously differentiable on $[T, \infty)$ and $\rho(t)>0, \rho^{\prime}(t)<0, \lim _{t \rightarrow \infty} \rho(t)=0$.

Let $u(t)$ be the solution of (4) on $[T, \infty)$ satisfying $u(T)=0$. Then $\lim \phi(t)=\infty[$ or $-\infty]$ implies $\lim u(t)=\infty$ [or $-\infty]$. $t \rightarrow \infty$ $t \rightarrow \infty$

LEMMA 2 [5]. Lèt $\sigma(t)$ be continuous on $[T, \infty)$ and let $v(t)$ be continuous differentiable on $[T, \infty)$. If the limit $\lim _{t \rightarrow \infty}\left[\sigma(t) v^{\prime}(t)+v(t)\right]$ exists in the extended real line $R^{\#}$, then the limit $\lim _{t \rightarrow \infty} v(t)$ exists in 
$R^{\#}$

THEOREM 1. Let the condition (3) hold. Suppose that $a(t) \geq 0$. If

$$
\int_{n-1}^{\infty} \rho_{n}(t) a(t) d t=\infty
$$

$$
\int^{\infty}|b(t)| d t<\infty \text {, }
$$

then alz nonoscizlatory solutions of (1) tend to zero as $t \rightarrow \infty$.

Proof. Let $y(t)$ be a nonoscillatory solution of (1). We may suppose that $y(g(t))>0$ for $t \geq t_{1}$. We define

$$
\begin{aligned}
G_{0}(t) & =y(t), G_{i}(t)=r_{i}(t) G_{i-1}^{\prime}(t), i=1, \ldots, n-1, \\
u_{k-1}(t) & \equiv \int_{t_{1}}^{t} \rho_{n-k}(s) G_{n-k}^{\prime}(s) d s \text { for } k=1,2, \ldots, n,
\end{aligned}
$$

which implies

$$
u_{k-1}(t)=-\frac{\rho_{n-k}(t)}{\rho_{n-k}^{\prime}(t)} u_{k}^{\prime}(t)+u_{k}(t)-\rho_{n-k}\left(t_{1}\right) G_{n-k}\left(t_{1}\right) .
$$

This shows that $u_{k}(t)$ satisfies the differential equation

$$
\frac{\rho_{n-k}(t)}{\rho_{n-k}^{\prime}(t)} u^{\prime}-u+\phi_{k}(t)=0
$$

or equivalently,

$$
u^{\prime}-\frac{\rho_{n-k}^{\prime}(t)}{\rho_{n-k}(t)} u+\frac{\rho_{n-k}^{\prime}(t)}{\rho_{n-k}(t)} \phi_{k}(t)=0,
$$

where

$$
\phi_{k}(t)=u_{k-1}(t)+\rho_{n-k}\left(t_{1}\right) G_{n-k}\left(t_{1}\right) .
$$

Since $u_{k}\left(t_{1}\right)=0$ by $(8)$ and since $\rho_{n-k}(t)>0, \rho_{n-k}^{\prime}(t)<0$, $\lim _{t \rightarrow \infty} \rho_{n-k}(t)=0$ by $(2 c)$, we apply Lemma 1 to $(10)$ to conclude that $\lim _{t \rightarrow \infty} u_{k-1}(t)=\infty[$ or $-\infty]$ implies that $\lim _{t \rightarrow \infty} u_{k}(t)=\infty[$ or $-\infty]$. Moreover, 
applying Lemma 2 to (9), we conclude that $\lim _{t \rightarrow \infty} u_{k}(t)$ exists in $R^{\#}$ whenever $\lim _{t \rightarrow \infty} u_{k-1}(t)$ exists in $R^{\#}$. From (1) we obtain

$$
G_{n-1}(t)-G_{n-1}\left(t_{1}\right)+\int_{t_{1}}^{t} a(s) f(y(g(s))) d s=\int_{t_{1}}^{t} b(s) d s .
$$

Since the first integral of (11) is positive and, by (6), the second integral is bounded, there exist a constant $K_{n-1}$ such that

$$
G_{n-1}(t)=r_{n-1}(t) G_{n-2}^{\prime}(t) \leq K_{n-1} \text { for } t \geq t_{2} \geq t_{1} \text {. }
$$

Dividing the inequality by $r_{n-1}(t)$ and integrating from $t_{2}$ to $t$, we get

$$
G_{n-2}(t)-G_{n-2}\left(t_{1}\right) \leq K_{n-1} \int_{t_{2}}^{t} \frac{d s}{r_{n-1}(s)} \text { for } t \geq t_{2} \text {, }
$$

which shows, in view of (3), that there exists a constant $K_{n-2}$ such that

$$
G_{n-2}(t)=r_{n-2}(t) G_{n-3}^{\prime}(t) \leq K_{n-2} \text { for } t \geq t_{2} \text {. }
$$

Applying the above argument repeatedly, we have

$$
G_{n-3}(t) \leq K_{n-3}, \cdots, G_{1}(t) \leq K_{1}, G_{0}(t) \leq K_{0} \text { for } t \geq t_{2},
$$

where $K_{n-3}, \ldots, K_{1}, K_{0}$ are constants. It follows that $G_{0}(t) \equiv y(t)$ is bounded above for $t \geq t_{2}$. We now multiply both sides of $(1)$ by $\rho_{n-1}(t)$ and integrate it over $\left[t_{2}, t\right]$. Then we have

$$
\begin{aligned}
\int_{t_{2}}^{t} \rho_{n-1}(s) G_{n-1}^{\prime}(s) d s+\int_{t_{2}}^{t} \rho_{n-1}(s) a(s) f(y(g(s))) d s & \\
& =\int_{t_{2}}^{t} \rho_{n-1}(s) b(s) d s .
\end{aligned}
$$

Noting that on account of (6) the right hand of (12) tends to a finite limit as $t \rightarrow \infty$, we can deduce from (12) that 


$$
\int_{t_{2}}^{\infty} \rho_{n-1}(t) a(t) f(y(g(t))) d t<\infty,
$$

since otherwise we could use Lemma 1 to obtain $\lim _{t \rightarrow \infty} u_{k}(t)=-\infty$ for $k=0,1, \ldots, n-1$, which implies $\lim _{t \rightarrow \infty} y(t)=-\infty$, a contradiction. Next, using (12), (13), the boundedness of $y(t)$ and applying Lemma 2, we can find that $\lim _{t \rightarrow \infty} u_{k}(t)$ is finite for each $k=0,1, \ldots, n-1$. Thus we see that $\lim _{t \rightarrow \infty} y(t)$ exists and finite. Namely, $\lim _{t \rightarrow \infty} y(t)=c$, where $c$ is a finite nonnegative constant. If $c>0$, then we have $c / 2 \leq y(g(t)) \leq 2 c$ for sufficiently large $t$, say $t \geq t_{3} \geq t_{2}$. From (13) and $f(y)$ is continuous, we have a contradiction that

$$
\infty>\int_{t_{3}}^{\infty} \rho_{n-1}(t) a(t) f(y(g(t))) d t \geq k^{*} \int_{t_{3}}^{\infty} \rho_{n-1}(t) a(t) d t=\infty,
$$

where

$$
K^{*}=\operatorname{Min}_{(c / 2) \leq y \leq 2 c} f^{\prime}(y)>0
$$

Therefore, we conclude that $y(t)$ tends to zero as $t \rightarrow \infty$.

REMARK. Kusano and Onose [5] obtain the same conclusion with the additional assumption $\lim \inf f(y)>0$ and $\lim \sup f(y)<0$.

$$
y \rightarrow \infty \rightarrow+\infty
$$

THEOREM 2. Let the condition (3) hold. Suppose that $a(t) \geq 0$, $\lim$ inf $f(y)>0$ and $\lim \sup f(y)<0$. If $y \rightarrow+\infty$

$$
\begin{gathered}
\int_{n-1}^{\infty} \rho_{n}(t) a(t) d t=\infty, \\
\int_{n-1}^{\infty} \rho_{n}(t)|b(t)| d t<\infty,
\end{gathered}
$$

then all nonoscilzatory solutions of (1) tends to zero as $t \rightarrow \infty$.

Proof. Let $y(t)$ be a nonoscillatory solution of (1). We may suppose that $y(g(t))>0$ for $t \geq t_{1}$. Define $G_{i}(t)$ and $u_{k}(t)$ by (7) 
and (8). We now multiply both sides of (1) by $\rho_{n-1}(t)$ and integrate it over $\left[t_{1}, t\right]$. Then we have

$$
\begin{aligned}
\int_{t_{1}}^{t} \rho_{n-1}(s) G_{n-1}^{\prime}(s) d s+\int_{t_{1}}^{t} \rho_{n-1}(s) a(s) f(y(g(s))) d s & \\
& =\int_{t_{1}}^{t} \rho_{n-1}(s) b(s) d s
\end{aligned}
$$

By using (12) and (15) we can deduce that

$$
\int_{t_{1}}^{\infty} \rho_{n-1}(t) a(t) f(y(g(t))) d t<\infty
$$

since otherwise we could use Lemma I to obtain $\lim _{t \rightarrow \infty} y(t)=-\infty$, a contradiction. Next, using (12), (13) and applying Lemma 2, we can find that $\lim _{t \rightarrow \infty} u_{k}(t) \quad(k=0,1, \ldots, n-1)$ exist as definite limit finite or $\infty$. Thus we see $\lim _{t \rightarrow \infty} y(t)=\infty$ or $\lim _{t \rightarrow \infty} y(t)=c$, where $c$ is a finite and nonnegative constant. If $\lim _{t \rightarrow \infty} y(t)=\infty$, then we have $\underset{t \rightarrow \infty}{\lim \inf } f(y(g(t)))>0$ by assumption, which and (14) lead to a contradiction that $\int_{t_{1}}^{\infty} \rho_{n-1}(t) a(t) f(y(g(t))) d t=\infty$.

If $\lim _{t \rightarrow \infty} y(t)=c>0$, then also we have a contradiction:

$$
\int_{t}^{\infty} \rho_{n-1}(t) a(t) f(y(g(t))) d t=\infty
$$

Therefore we conclude that $y(t)$ tends to zero as $t \rightarrow \infty$. //

REMARK. Theorem 2 contains the result of Kusano and Onose ([5], Theorem 3).

EXAMPLE 1. Consider the equation

$$
\left(t^{2}\left(t^{2}\left(t^{2} y^{\prime}(t)\right)^{\prime}\right)^{\prime}\right)^{\prime}+t^{7} y^{3}(\gamma t)=\gamma^{-6} t, \quad t>0,
$$


where $\gamma$ is a positive constant. In this case we have $\rho_{1}(t)=t^{-1}$, $\rho_{2}(t)=(1 / 2) t^{-2}, \rho_{3}(t)=(1 / 6) t^{-3}$. Since all assumptions of Theorem 2 are satisfied, every nonoscillatory solution of (16) approaches zero as $t \rightarrow \infty$. This equation has a nonoscillatory solution $y(t)=t^{-2}$.

EXAMPLE 2. Consider the equation

(17) $\left(e^{t}\left(e^{t}\left(e^{t} y^{\prime}(t)\right)^{\prime}\right)^{\prime}\right)^{\prime}+e^{5 t} y(t+\theta)=24 e^{-t}+e^{-4 \theta} e^{t}, \quad t \geq 0$,

where $\theta$ is a constant. This equation possesses $y(t)=e^{-4 t}$ as a nonoscillatory solution tending to zero as $t \rightarrow \infty$. It is easy to verify that $\rho_{1}(t)=e^{-t}, \rho_{2}(t)=(1 / 2) e^{-2 t}, \rho_{3}(t)=(1 / 6) e^{-3 t}$ and the conclusions of Theorem 2 are satisfied. Therefore all nonoscillatory solutions of (17) also tend to zero as $t \rightarrow \infty$.

REMARK. These examples cannot be covered by Kusano and Onose ([5], Theorem 3).

\section{References}

[1] John R. Graef and Paul W. Spikes, "Asymptotic behavior of solutions of a second order nonlinear differential equation", $J$. Differential Equations 17 (1975), 461-476.

[2] R. Grimmer, "On nonoscillatory solutions of a nonlinear differential equation", Proc. Amer. Math. Soc. 34 (1972), 118-120.

[3] Michael E, Hammett, "Nonoscillation properties of a nonlinear differential equation", Proc. Amer. Math. Soc. 30 (1971), 92-96.

[4] Athanassios G. Kartsatos, "Oscillation and existence of unique positive solutions for nonlinear $n$th order equations with forcing term", Hiroshima Math. J. 6 (1976), 1-6.

[5] Takaŝi Kusano and Hiroshi Onose, "Nonoscillation theorems for differential equations with deviating argument", Pacific J. Math. 63 (1976), 185-192. 
[6] Takaŝi Kusano and Hiroshi Onose, "Asymptotic behavior of nonoscillatory solutions of second order functional differential equations", BulZ. Austral. Math. Soc. 13 (1975), 291-299.

[7] Stig-Olof Londen, "Some nonoscillation theorems for a second order nonlinear differential equation", SIAM J. Math. Anal. 4 (1973), $460-465$.

[8] Bhagat Singh, "Asymptotic nature of nonoscillatory solutions of $n$th order retarded differential equations", SIAM J. Math. Anal. 6 (1975), 784-795.

Department of Mathematics,

Ibaraki University,

Mito 310 ,

Japan. 